\title{
Introduction to Special Issue on Future of Wireless Communication and 6G Networks
}

\author{
Mamoun Alazab ${ }^{1} \cdot$ Ameer Al-Nemrat $^{2} \cdot$ Mohammad Shojafar $^{3} \cdot{\text { Xinrong } \mathrm{Li}^{4}}^{4}$ \\ Accepted: 30 July 2021 / Published online: 18 August 2021 \\ (c) The Author(s), under exclusive licence to Springer Science+Business Media, LLC, part of Springer Nature 2021
}

Wireless communication is the fastest and efficient communication model, enabling us to connect across the globe anytime. Wireless technologies are proliferating at a substantial rate allowing people to communicate even from rural regions making their lives better. The existing network that we use today consumes high data and battery usage, which restricts users with limited internet usage. Hence, future generation needs a network with reliable speed and wide data coverage. Definitely, 6G networks are expected to resolve these issues and revolutionize the globe with advanced systems for faster and effective communication. This special issue brings together researchers from various backgrounds to discuss the $6 \mathrm{G}$ network that integrates artificial intelligence and the internet of space things for super-advanced space communication of unidentified aerial objects. In parallel, this special issue also encourages the researchers and practitioners to share insights on the future of $6 \mathrm{G}$ networks and their expansion in various fields like imaging, space technology and ultra-speed sampling techniques, deep learning, and big data analytics. The primary objective of this special section is to

Mamoun Alazab

m.alazab@ieee.org

Ameer Al-Nemrat

a.al-nemrat@uel.ac.uk

Mohammad Shojafar

m.shojafar@surrey.ac.uk

Xinrong Li

Xinrong.Li@unt.edu

1 IT and Environment, Charles Darwin University, Casuarina, NT, Australia

2 Faculty in School of Architecture Computing and Engineering, University of East London, Newham, London, UK

3 Institute for Communication Systems (ICS), University of Surrey, Guildford, UK

4 Department of Electrical Engineering, University of North Texas, Denton, TX, USA understand features of $6 \mathrm{G}$ networks like speed, data transfer and storage.

This special section includes eight articles, the first article deals with optimal regression sampling estimation methods for wireless communication technology to improve performance and shortcomings of the existing sampling survey methods. The second article presents a wireless sensor network communication technology to improve the performance of the composition rule perception algorithm of the national art plane system. The third article presents a distance teaching platform network for English courses designed for communication engineering, innovatively combining network technology with the education field. The fourth article focuses on using the evaluation index on machine learning for network intrusion detection. This paper benefits from the principle of structural risk minimization to pre-process the data, learn and train the data, calculate important features of data, and reduce the data dimension. Subsequently, the technique presented in the paper increases accuracy and efficiency and effectively reduce the false alarm rate for network intrusion detection.

The fifth paper in the series is an article on designing an online monitoring system of distribution transformer collaborating cloud and Internet of Things for reducing long response time and improved performance. In the sixth paper the author presents a situational English language information intelligent retrieval algorithm based on a wireless sensor network model and communication routing mechanism for shortening the retrieval time and accuracy improvement. The seventh article in this special issue presents a virtual threedimensional model construction method based on mobile communication technology. This technology not only helps in transforming and updating the ageing residential buildings but also make them concise, real, and energy saving. Finally, the eighth article introduces the power corridor visualization technology for UAV line inspection data in carrying out visualization processing through data acquisition level image denoising. This approach in turn improves the 
accuracy of UAV line inspection and ensure the safety of the power system.

The response from the research community for this special issue was significant, and all the articles that are approved for publication have undergone a double-blinded review process to meet the standards of the journal. We ensure that this special issue interprets the importance and need of $6 \mathrm{G}$ networks for future wireless communication systems, which will revolutionize the entire communication medium with its advances in security and efficiency. We are very much grateful to all the authors for their innovative contributions and all the reviewers for their timely efforts. Finally, we thank the Editor-in-Chief for offering us the privilege to edit this special issue in this reputed journal. Three more papers related to this SI will appear in the next issue of the journal.

Publisher's Note Springer Nature remains neutral with regard to jurisdictional claims in published maps and institutional affiliations.

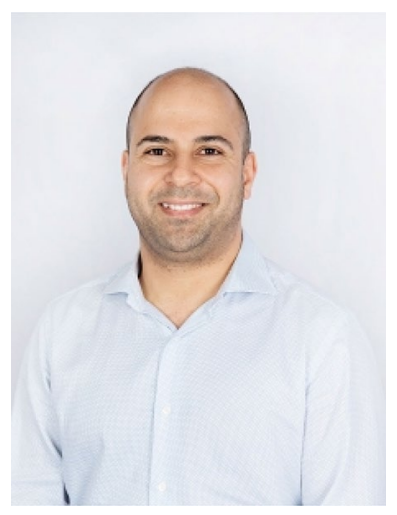

Mamoun Alazab is an Associate Professor at the College of Engineering, IT and Environment at Charles Darwin University, Australia. He received his $\mathrm{PhD}$ degree in Computer Science from the Federation University of Australia, School of Science, Information Technology and Engineering. He is a cyber security researcher and practitioner with industry and academic experience. Alazab's research is multidisciplinary that focuses on cyber security and digital forensics of computer systems with a focus on cybercrime detection and prevention. He has more than 150 research papers in many international journals and conferences, such as IEEE transactions on Industrial Informatics, IEEE Transactions on Industry Applications, IEEE Transactions on Big Data, IEEE Transactions on Vehicular Technology, Computers \& Security, and Future Generation Computing Systems. He delivered many invited and keynote speeches, 24 events in 2019 alone. He convened and chaired more than 50 conferences and workshops. He works closely with government and industry on many projects, including Northern Territory (NT) Department of Information and Corporate Services, IBM, Trend Micro, the Australian Federal Police (AFP), the Australian Communications and Media Authority (ACMA), Westpac, United Nations Office on Drugs and Crime (UNODC), and the Attorney General's Department. He is a
Senior Member of the IEEE. He is the Founding chair of the IEEE Northern Territory (NT) Subsection.

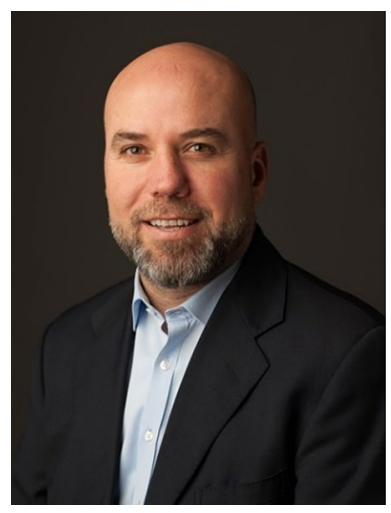

Ameer Al-Nemrat is working as a Senior Lecturer in School of Architecture, Computing and Engineering, University of East London (UEL), he is a Director of Professional Doctorate in Information Security \& the MSc Information Security and Digital Forensics programmes. He is a founder and director of the Electronic Evidence Laboratory. In addition, he is a co-editor of the book "Issues in Cybercrime, Security, and Digital Forensics". $\mathrm{He}$ worked in the project of Cybercrime Programme Project with a German Institution. He was nominated as a Best Lecturer for Student Led Teaching Awards in 2015. His working experience in designing and leading information security projects and programmes, His research areas also include the cybercrime and digital forensics, cyber-criminal network analysis using data mining techniques.

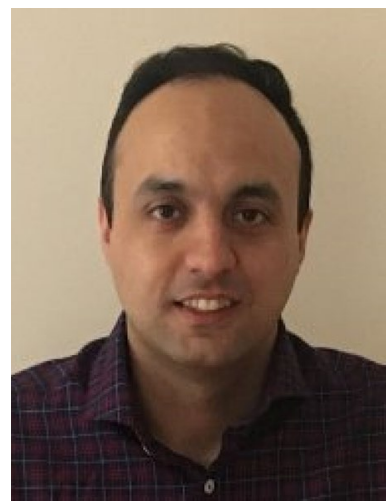

Mohammad Shojafar is an Associate Professor in the Institute for Communication Systems (ICS) at the University of Surrey, United Kingdom. He received a Ph.D. in ICT from Sapienza University of Rome, Italy. He received the honored B.Sc. in CS at Iran University Science and Technology, Tehran, Iran in 2006. He was a programmer/analyzer at the National Iranian Oil Company (NIOC) and Tidewater Limited in Iran from 2008-2013, respectively. He published over 100 refereed top-tier articles in prestigious venues such as IEEE TCC, IEEE TNSM, IEEE Network, Computer Networks, and FGCS. He is an Associate Editor in IEEE Transactions on Consumer Electronics, IET Communications, Cluster Computing, and Ad Hoc \& Sensor Wireless Networks Journals. He published a book entitled "Cybersecurity and Privacy in Cyber Physical Systems" which is appeared in CRC Taylor and Francis, recently. He is also an Intel Innovator and a IEEE Senior Member. His research interests are Network Security, Cryptography algorithms, Green Networking, Cloud and Fog Networks, Distributed and Networked Systems. 


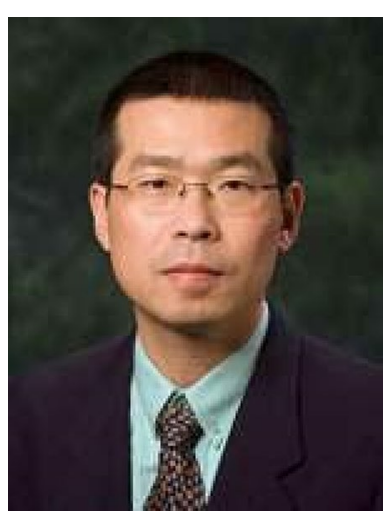

Xinrong Li is an Associate Professor and the Associate Department Chair at the University of North Texas. His general area of research is in wireless access and localization for the emerging cyberspace applications. He has published well cited papers in the field of indoor geolocation. $\mathrm{He}$ has received his BS from the University of Science and Technology, Hefei, China (1995), MS degree from the National University of Singapore (1999), and PhD from the Worcester
Polytechnic Institute, Worcester, MA (2003). He is a senior member of IEEE Communications Society and an editor of the International Journal of Wireless Information Networks, Springer Nature. He has also played administrative roles in numerous IEEE conferences such as PIMRC and ISMICT. 